\title{
LPS-induced reduction of triglyceride synthesis and secretion in dairy cow mammary epithelial cells via decreased SREBP1 expression and activity
}

\author{
Jianfa Wang ${ }^{1,2 *}$, Xu Zhang ${ }^{1,2}$, Xianjing He $\mathrm{H}^{1,2}$, Bin Yang ${ }^{1,2}$, Hai Wang ${ }^{1,2}$, Xufei Shan ${ }^{1,2}$, Chunqiu Li ${ }^{1,2}$, \\ Dongbo Sun ${ }^{1,2}$ and Rui $\mathrm{Wu}^{1,2 *}$ \\ 'College of Animal Science and Veterinary Medicine, Heilongjiang Bayi Agricultural University, Daqing 163319, China \\ ${ }^{2}$ Heilongjiang Provincial Key Laboratory of Prevention and Control of Bovine Diseases, Daqing 163319, China
}

Received 13 December 2017; accepted for publication 15 June 2018; first published online 8 August 2018

\begin{abstract}
Sterol regulatory element binding protein 1 (SREBP1) has a central regulatory effect on milk fat synthesis. Lipopolysaccharides (LPS) can induce mastitis and cause milk fat depression in cows. SREBP1 is also known to be associated with inflammatory regulation. Thus, in the current study, we hypothesized that LPS-induced milk fat depression in dairy cow mammary epithelial cells (DCMECs) operates via decreased SREBP1 expression and activity. To examine the hypothesis, DCMECs were isolated and purified from dairy cow mammary tissue and treated with LPS $(10 \mu \mathrm{g} / \mathrm{ml})$. LPS treatment of DCMECs suppressed lipid-metabolism-related transcription factor SREBP1 mRNA expression, nuclear translocation and protein expression, leading to reduced triglyceride content. The transcription levels of acetyl-CoA carboxylase- 1 and fatty acid synthetase were significantly down-regulated in DCMECs after LPS treatment, suggesting that acetyl-CoA carboxylase- 1 and fatty acid synthetase involved in de novo milk fat synthesis was regulated by SREBP1. In summary, these results suggest that LPS induces milk fat depression in dairy cow mammary epithelial cells via decreased expression of SREBP1 in a time-dependent manner.
\end{abstract}

Keywords: Dairy cow mammary epithelial cells, lipopolysaccharides, sterol regulatory element binding protein 1 , milk fat synthesis.

Many researchers have investigated the synthesis and regulation mechanism of milk fat, because milk fat is important for the nutritional quality and flavor of milk. Milk fat consists predominantly of triglyceride (TG; $>95 \%$ ), diglyceride $(2 \%)$, phospholipids (1\%), cholesterol $(0.5 \%)$ and small amount of free fatty acids (FFA) $(\sim 0 \cdot 1 \%)$ in all mammals (Staniewski et al. 2012). Milk fat is the major material basis for the nutritional quality in milk, which can provide nutrition and energy to human beings. Mammary epithelial cells can synthesize and secrete FA. There are two main sources of milk FA: short chain (4-8 C) and medium chain (10-14 C), and a portion of 16-C FA that arise de novo almost exclusively from DCMECs using acetic acid, $\beta$-hydroxybutyric acid and glycerol as precursor substances that are catalyzed by acetyl-CoA carboxylase-1 (ACC1) and fatty acid synthase (FAS). Remaining 16-C FA and long chain FA (>16 C) are

*For correspondence; e-mail: wjflw@sina.com; fuhewu@126.com obtained from the blood of cows and are produced by DCMECs using lipoprotein lipase (LPL), acetyl-CoA binding protein (ACBP), CD36 and other FA transport enzymes (Bionaz and Loor, 2008). Lipid-metabolismrelated transcription factor sterol regulatory element binding protein 1 (SREBP1) regulates the synthesis of dairy cow milk fat by regulating expression of the above-mentioned FA-synthesizing genes, SREBP1 plays an important role in milk fat uptake, transport, and de novo synthesis (Ma, 2012; Rincon et al. 2012).

Lipopolysaccharides (LPS) from the cell wall of Gramnegative bacteria are also referred to as a bacterial endotoxin. Bacteria will release LPS during clinical disease such as ruminal acidosis, mammary and uterine infection as well as during heat stress (Gott, 2011). LPS is harmful to dairy cows, causing systemic and local inflammatory reactions, and leading to a serious decrease in milk fat percentage and yield, and consequent major economic losses in the dairy farming and processing industries. It is known that LPS can reduce the content of milk fat synthetic precursors in the 
blood. LPS also induces acute phase reactions of the liver, resulting in damage to the liver that then does not provide sufficient amounts of apolipoproteins required for VLDL assembly. Since VLDL particles are an important source of fatty acids for milk fat production, LPS-induced acute phase reactions of the liver can lead to a reduction in milk fat synthesis.

In addition, meta-analysis of ruminant mastitis differential gene expression spectra has indicated that SREBP1 may be involved in the adaptive response to mammary infection (Genini et al. 2011). SREBP1 also has a role in inflammatory regulation, and has been attracting increased research attention (Spann et al. 2012; Wei and Espenshade, 2012; Oishi et al. 2017). It is unclear whether LPS can regulate the expression and activity of SREBP1, and hence influence the synthesis of milk fat in DCMECs. Thus, in the current study, we hypothesized that LPS-induced milk fat depression in DCMECs operates via decreased SREBP1 expression and activity. These results will provide a scientific basis to study further the regulatory mechanism of milk fat synthesis and improve the nutritional quality of cow's milk.

\section{Materials and methods}

\section{Isolation of DCMECs and LPS treatment}

Primary DCMECs were cultured and purified as described previously (Xu et al. 2017). Experiments were performed using the 5th passage DCMECs. Forty-eight hours before treatment, insulin and hydrocortisone concentrations were reduced to $0 \%$, and the concentration of other components was unchanged. LPS (Escherichia coli 055:B5; $10 \mu \mathrm{g} / \mathrm{ml}$; Sigma) was added to the new medium. DCMECs were treated with LPS and collected for subsequent analysis. Experiments were repeated three times.

\section{Real-time quantitative reverse transcription polymerase chain reaction ( $q R T-P C R$ )}

Total RNA from DCMECs was extracted with TRIzol reagent (Sigma). The concentration and mass of RNA were measured at 260/280 nm using an ultraviolet spectrophotometer (Thermo Fisher Scientific, Waltham, MA, USA). RNA with $\mathrm{OD}_{260 / 280} 1 \cdot 8-2 \cdot 0$ was reverse-transcribed to cDNA using PrimeScript RT Reagent Kit with gDNA Eraser (TaKaRa Biotechnology, Tokyo, Japan). qRT-PCR primers were designed using Primer 5.0 software (Applied Biosystems, Foster City, CA, USA; sequences are given in online Supplementary Table S1). qRT-PCR was performed using the Bio-Rad CFX96 Real-Time PCR Detection System (Bio-Rad, Hercules, CA, USA), and SYBR Premix Ex Taq (TaKaRa Biotechnology). Glyceraldehyde-3-phosphate dehydrogenase (GAPDH) was used as a reference gene (Wu et al. 2015). The relative expression level of genes was calculated by normalizing to GAPDH using the $2^{-\Delta \Delta \mathrm{Ct}}$ method (Huang et al. 2012; Li et al. 2014).

\section{Western blotting}

Western blotting for SREBP1 in DCMECs was performed as described previously (Liu et al. 2016; Song et al. 2016). Primary antibodies: mouse anti-SREBP1 monoclonal antibody (ab3259; $1: 1000$ dilution; Abcam); rabbit anti- $\beta$-actin antibody (bs-0061R; $1: 2000$ dilution; Bioss, Woburn, MA, USA). Secondary antibodies: horseradish peroxidase (HRP)-labeled goat anti-mouse $\operatorname{lgG}(\mathrm{H}+\mathrm{L})(\mathrm{ab6789} ; 1: 5000$ dilution; Abcam); HRP-labeled goat anti-rabbit $\operatorname{lgG}(\mathrm{H}+\mathrm{L})$ (ab205718; 1:5000 dilution; Abcam). The ECL signal was detected using a gel imaging system, and OD values of western blotting images were analyzed using Image-Pro Plus 6.0 software (IPP; Media Cybernetics, Bethesda, MD, USA).

\section{Nuclear translocation assay of SREBP1}

Nuclear translocation for SREBP1 in DCMECs was performed as described previously (Huang et al. 2013). Primary antibodies: mouse anti-SREBP1 monoclonal antibody (ab3259; $1: 1000$ dilution; Abcam). Secondary antibodies: Alexa Fluor 488-labeled goat anti-mouse $\operatorname{lgG}$ (ab150117; $1: 500$ dilution; Abcam). The coverslips were photographed using laser confocal microscopy (Leica).

\section{BODIPY493/503 staining of lipid droplets}

Staining for lipid droplets in DCMECs was performed as described previously (Lee et al. 2012).

\section{Determination of TG content}

The total protein concentration of cells was determined by the Enhanced BCA Protein Assay Kit (Pierce, Rockford, IL, USA). TG concentration of the DCMECs was quantification using the Tissue Triglyceride Assay Kit (Applygen Technologies, Beijing, China) at $550 \mathrm{~nm}$. The TG concentration of DCMECs was corrected to concentration per mg protein (He et al. 2012; Shi et al. 2016).

\section{Statistical analysis}

Each sample was assessed in triplicate and the experimental data were expressed as the mean \pm SD. The data were analyzed by one-way analysis of variance using SPSS version $19 \cdot 0$ software (SPSS, IBM, Armonk, NY, USA). The differences were considered significant at $P<0.05$ and highly significant at $P<0 \cdot 01$.

\section{Results}

LPS decreases transcription, translation and nuclear translocation of SREBP1

The mRNA expression level of SREBP1 was reduced in DCMECs treated with LPS for $3 \mathrm{~h}$, and gradually decreased further with LPS treatment time $(P<0.01$ or $P<0 \cdot 05)$. Transcription of SREBP1 was lowest at $12 \mathrm{~h}$ after LPS 
(a)

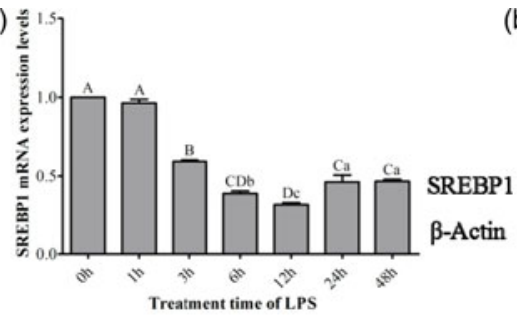

(b)

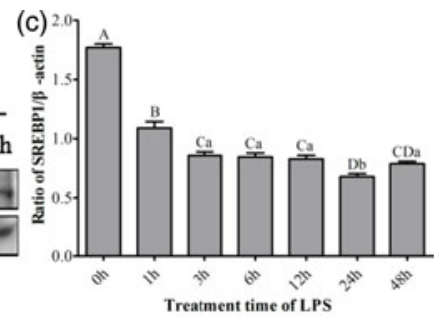

Treatment time of LPS

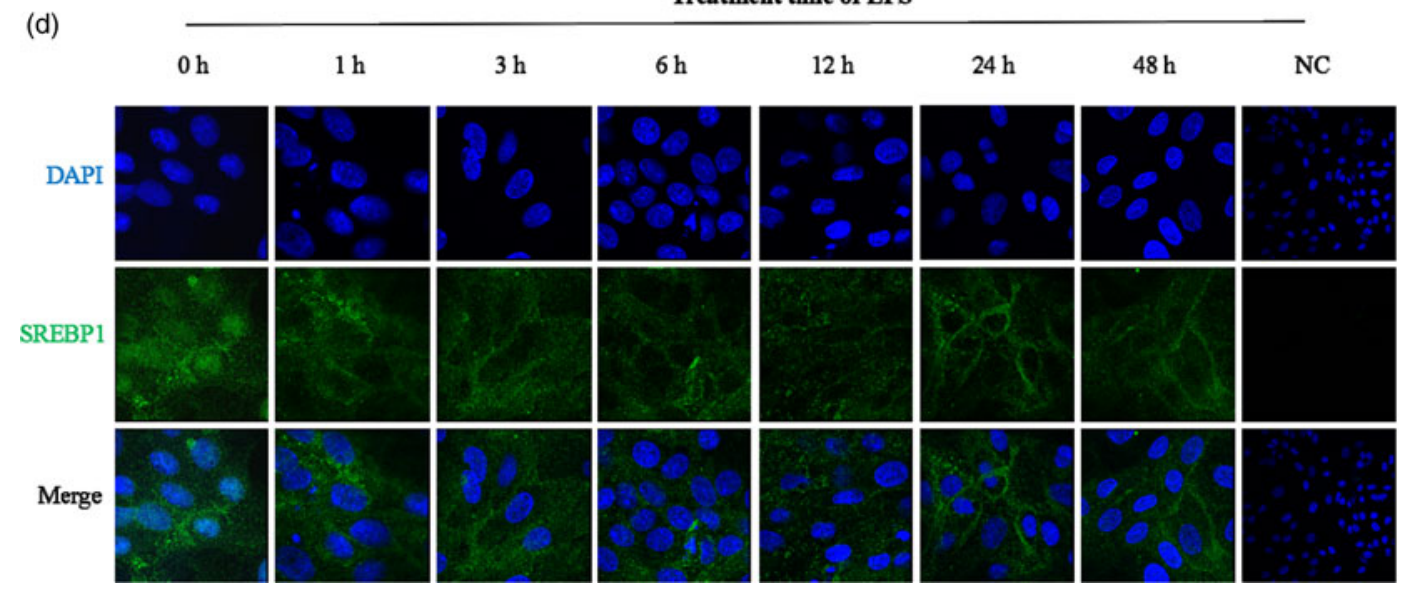

Fig. 1. Transcription, translation and nuclear translocation of SREBP1 in DCMECs. (a) Transcription levels of SREBP1. (b) Western blotting of SREBP1. (c) The grayscale analysis of SREBP1. (D) Nuclear translocation assay of SREBP1. Note: The data with different capital letters between two groups showed very significant differences $(P<0 \cdot 01)$; data with different lower-case letters between two groups showed significant differences $(P<0 \cdot 05)$; data with the same letters between two groups showed no significant differences $(P>0 \cdot 05)$.

treatment, and then began to increase gradually $(P<0 \cdot 01)$. However, the trend for increased SREBP1 transcription after LPS treatment for 24 and $48 \mathrm{~h}$ was not obvious, and the difference was not significant $(P>0 \cdot 05)$ (Fig. 1a).

Western blotting revealed that protein expression of SREBP1 was down-regulated in DCMECs treated with LPS for $1 \mathrm{~h}$, and gradually decreased further with LPS treatment time $(P<0 \cdot 01)$. Expression of SREBP1 protein was lowest at $24 \mathrm{~h}$ after LPS treatment, and then began to increase gradually $(P<0.05)$ (Fig. $1 \mathrm{~b}, \mathrm{c})$.

Immunofluorescence revealed that after treatment with LPS for $1 \mathrm{~h}$, the intracellular trafficking of mature SREBP1 to the nucleus was inhibited. The levels of intracellular trafficking of mature SREBP1 to the nucleus were inhibited least at $24 \mathrm{~h}$ after LPS treatment, and then began to increase gradually (Fig. 1d). These results suggest that treatment with LPS decreases expression and activity of SREBP1 in DCMECs in a time-dependent manner.

\section{LPS decreases transcription levels of FAS and ACC1}

Transcription of FAS was down-regulated in DCMECs treated with LPS for $3 \mathrm{~h}$, and gradually decreased further with LPS treatment time $(P<0 \cdot 01)$. Transcription of FAS was lowest at $24 \mathrm{~h}$ after LPS treatment, and then began to increase gradually $(P<0 \cdot 01)$ (Fig. 2a). The transcriptional changes of ACC1 are consistent with those of FAS (Fig. 2b).

\section{LPS decreases milk fat synthesis}

The content of TG was decreased in DCMECs treated with LPS for $3 \mathrm{~h}$, and gradually decreased further with LPS treatment time $(P<0.01$ or $P<0.05)$. The content of lipid droplets was lowest at $12 \mathrm{~h}$ after LPS treatment, and then began to increase gradually $(P<0 \cdot 05)$ (Fig. 3). However, the trend for increased milk fat synthesis after LPS treatment for 24 and $48 \mathrm{~h}$ was not obvious and the difference was not significant $(P>0 \cdot 05)$. The results of the lipid droplets BODIPY493/503 staining was consistent with the results of the determination of TG content (data are given in online Supplementary Fig. S1). These results suggest treatment with LPS can decrease milk fat synthesis in DCMECs in a time-dependent manner.

\section{Discussion}

SREBP1 belongs to the basic helix-loop-helix-leucine zipper family of transcription factors, and is essential for the regulation of $\mathrm{FA}$ and cholesterol biosynthetic gene expression (Jeon and Osborne, 2012). Genome-wide association scans and functional genomics analyses have established that SREBP1 is a key regulator of milk fat synthesis and secretion (Ogorevc et al. 2009). It can regulate the synthesis of fat in the body by controlling the expression of the relevant lipid-synthesizing-related enzymes involved in the 

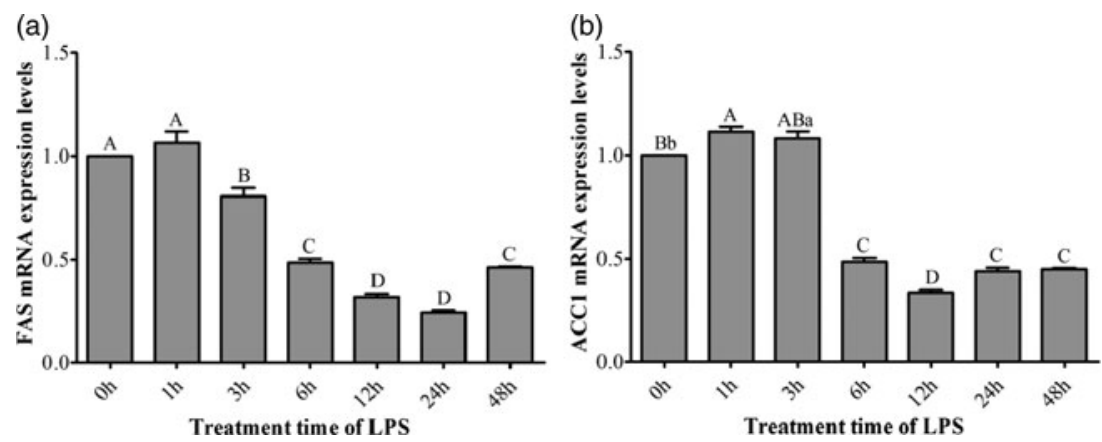

Fig. 2. Transcription levels of FAS and ACC1 in DCMECs. (a) Transcription levels of FAS. (b) Transcription levels of ACC1. Note: The data with different capital letters between two groups showed very significant differences $(P<0 \cdot 01)$; data with different lower-case letters between two groups showed significant differences $(P<0 \cdot 05)$; data with the same letters between two groups showed no significant differences $(P>0 \cdot 05)$.

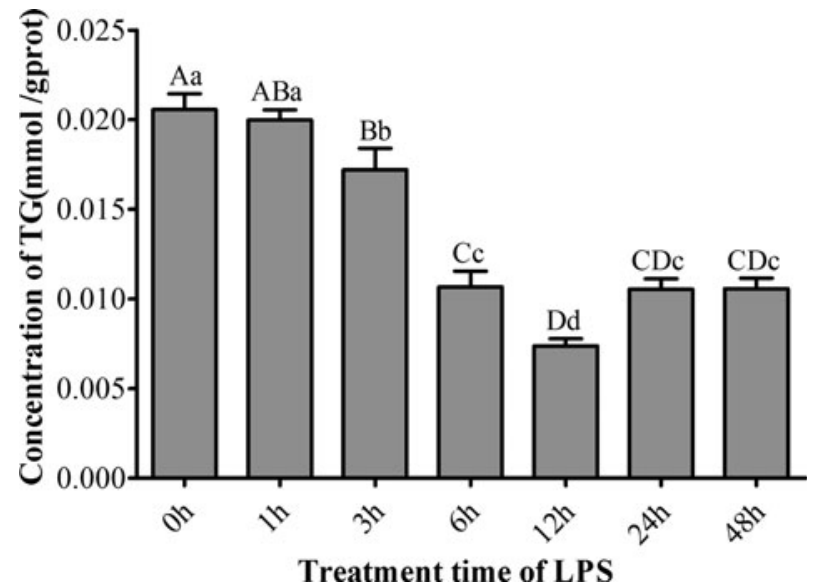

Fig. 3. Concentration of TG in DCMECs. Note: The data with different capital letters between two groups showed very significant differences $(P<0 \cdot 01)$; data with different lower-case letters between two groups showed significant differences $(P<$ 0.05); data with the same letters between two groups showed no significant differences $(P>0 \cdot 05)$.

synthesis and uptake of cholesterol, FA, TG and phospholipids. Normally, SREBP1 in the endoplasmic reticulum is an inactive precursor. When cells are stimulated by liver $X$ receptor, specificity protein 1 and oxysterols, SREBP1 is activated. Following activation, the amino terminal fragments of SREBP1 translocate to the nucleus and initiate transcription of target genes in combination with the SREs within the promoters of target genes (Zhang et al. 2015). Numerous cell culture experiments and genetically modified mouse models have shown that the major target genes of SREBP1 are some of the rate-limiting enzymes in the FA and cholesterol biosynthesis pathways. After treatment of DCMECs with SREBP1 siRNA, ACC1 and FAS content decreased by 40-65\% (Ma \& Corl, 2012). It has also been found that the mRNA expression levels of SREBP1 and milk fat synthesis enzymes in dairy cows are up-regulated in lactation, indicating that SREBP1 has a central regulatory effect on milk fat synthesis (Farke et al. 2008).
Milk fat synthesis involves the de novo synthesis of FA as well as the incorporation of de novo and preformed FA into TG. The TG accumulates to form lipid droplets, primarily in the mammary gland tissues of mammals. The activation of these metabolic pathways requires the coordinated regulation of a network of genes encoding lipogenic enzymes, such as the lipid-metabolism-related transcription factor SREBP1, as well as the de novo FA synthesis genes FAS and ACC1. FAS and ACC1 are the key enzymes for de novo synthesis of milk fat in DCMECs, and the gene promoter region of both has the binding site of SREBP1. Acetyl-CoA synthetase converts acetate into acetyl-CoA and begins de novo synthesis of milk fat. ACC1 catalyzes the conversion of acetyl-CoA to malonyl-CoA, a step that is the rate-limiting step in milk fat synthesis. FAS utilizes acetyl-CoA as a substrate to catalyze a series of carbon chain extension reactions to add the two carbon units from malonyl-CoA to the gradually elongated fatty acyl chain. SREBP1 translocates to the nucleus where it activates lipogenic genes by binding to the SREBP1 response element of the ACC1 and FAS genes (Kim et al. 2010). The mRNA transcription levels of ACC1 and FAS, as well as TG secretion and lipid droplets formation represent the DCMECs' capacity for milk fat synthesis. In this study, we found that SREBP1 affected the mRNA expression levels of the FAS and ACC1 genes in accordance with changes in TG content and lipid droplets accumulation, further confirming that SREBP1 acts on its target genes to regulate milk fat synthesis in DCMECs.

LPS is an important pathogen-associated molecular pattern that reduces the concentration of milk fat synthetic precursors in the blood of dairy cows, and can also be identified by pattern recognition receptors (e.g. Toll-like receptor 4) on the surface of mammary epithelial cells, resulting in up-regulated DNA binding activity of nuclear factor (NF)$\kappa \mathrm{B}$ and increased expression of inflammatory cytokines including TNF $\alpha$, IL-1 $\beta$, IL- 6 and IL- 8 . This creates a highly inflammatory cytokine state in the mammary tissue and even in the body as a whole (Miao et al. 2012). The binding site of NF-KB has been found in the promoter region of SREBP1 (Zhang et al. 2005). These results 
suggest that the inflammatory signaling pathway has the potential to regulate lipid metabolism.

In this study, LPS decreased the concentration of TG and the formation of lipid droplets in DCMECs, and decreased the transcriptional and nuclear translocation of lipogenic transcription factor SREBP1 in DCMECs. At the same time, LPS decreased the expression of milk fat de novo synthesis-related enzyme genes in DCMECs. Moreover, these results suggest that LPS affects the synthesis of dairy cow milk fat by down-regulating expression of SREBP1 and milk fat de novo synthesis of related enzyme genes. Nevertheless, expression of milk-fat-synthesis-related genes and proteins and TG is not always decreased after DCMECs are treated with LPS, but with the prolongation of treatment, there is a tendency for increased expression of milk-fat-synthesis-related genes and proteins and TG. This may be due to the fact that LPS is identified by the pattern recognition receptor on the surface of mammary epithelial cells to upregulate the DNA binding activity of NF- $\kappa B$, increase the transcription of SREBP1, and enable SREBP1 to participate in inflammatory regulation. However, this still needs validation. This may explain why cow mastitis can further aggravate the depression of milk fat.

In conclusion, our study suggests that LPS induces milk fat depression in DCMECs via decreased SREBP1 expression and activity in a time-dependent manner.

\section{Conflict of interests}

All the authors in this article claim no conflicts of interest.

\section{Supplementary material}

The supplementary material for this article can be found at https://doi.org/10.1017/S0022029918000547.

The present study was jointly financially supported by the National Natural Science Foundation of China $(31472249,31402157$, and 31772698), the Natural Science Foundation of Heilongjiang province (QC2016045), the Doctoral Fund of Ministry of Education of China (2016M601465), the University Nursing Program for Young Scholars with Creative Talents in Heilongjiang Province (UNPYSCT-2015087), and the Startup Foundation for the Doctors in Heilongjiang Bayi Agricultural University (XYB2014-12).

\section{References}

Bionaz M \& Loor JJ 2008 Gene networks driving bovine milk fat synthesis during the lactation cycle. BMC Genomics 9366

Farke C, Meyer HH, Bruckmaier RM \& Albrecht C 2008 Differential expression of $A B C$ transporters and their regulatory genes during lactation and dry period in bovine mammary tissue. Journal of Dairy Research 75 406-414

Genini S, Badaoui B, Sclep G, Bishop SC, Waddington D, Laan MH, Klopp C, Cabau C, Seyfert HM, Petzl W, Jensen K, Glass EJ, Greeff AD, Smith HE, Smits MA, Olsaker I, Boman GM, Pisoni G, Moroni P, Castiglioni B, Cremonesi P, Corvo MD, Foulon E,
Foucras G, Rupp R \& Giuffra E 2011 Strengthening insights into host responses to mastitis infection in ruminants by combining heterogeneous microarray data sources. BMC Genomics 12 315-316

Gott PN 2011 Endotoxin Tolerance in Lactating Dairy Cows. Columbus, USA: The Ohio State University

He YH, He Y, Liao XL, Niu YC, Wang G, Zhao C, Wang L, Tian MJ, Li Y \& Sun $\mathbf{C H} 2012$ The calcium-sensing receptor promotes adipocyte differentiation and adipogenesis through PPAR $\gamma$ pathway. Molecular and Cellular Biochemistry 361 321-328

Huang JG, Gao XJ, Li QZ, Lu LM, Liu R, Luo CC, Wang JL, Qiao B \& Jin X 2012 Proteomic analysis of the nuclear phosphorylated proteins in dairy cowmammary epithelial cells treated with estrogen. In Vitro Cellular and Developmental Biology-Animal 48 449-457

Huang YL, Zhao F, Luo CC, Zhang X, Si Y, Sun Z, Zhang L, Li QZ \& Gao XJ 2013 SOCS3-mediated blockade reveals major contribution of JAK2/ STAT5 signaling pathway to lactation and proliferation of dairy cow mammary epithelial cells in vitro. Molecules 18 12987-13002

Jeon TI \& Osborne TF 2012 SREBPs: metabolic integrators in physiology and metabolism. Trends in Endocrinology and Metabolism Tem 23 65-72

Kim YM, Shin HT, Seo YH, Byun HO, Yoon SH, Lee IK, Hyun DH, Chung HY \& Yoon G 2010 Sterol regulatory element-binding protein (SREBP)-1-mediated lipogenesis is involved in cell senescence. Journal of Biological Chemistry 285 29069-29077

Lee JS, Mendez R, Heng HH, Yang ZQ \& Zhang K 2012 Pharmacological ER stress promotes hepatic lipogenesis and lipid droplet formation. American Journal of Translational Research 4 102-113

Li N, Zhao F, Wei C, Liang M, Zhang N, Wang C, Li QZ \& Gao XJ 2014 Function of SREBP1 in the milk fat synthesis of dairy cow mammary epithelial cells. International Journal of Molecular Sciences 1516998 17013

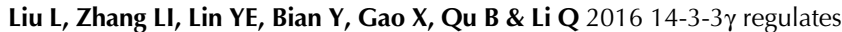
cell viability and milk fat synthesis in lipopolysaccharide-induced dairy cow mammary epithelial cells. Experimental and Therapeutic Medicine 11 1279-1287

Ma L \& Corl BA 2012 Transcriptional regulation of lipid synthesis in bovine mammary epithelial cells by sterol regulatory element binding protein-1. Journal of Dairy Science 95 3743-3755

Miao J, Fa Y, Gu B, Zhu W \& Zou S 2012 Taurine attenuates lipopolysaccharide-induced disfunction in mouse mammary epithelial cells. Cytokine 59 35-40

Ogorevc J, Kunej T, Razpet A \& Dovc P 2009 Database of cattle candidate genes and genetic markers for milk production and mastitis. Animal Genetics 40 832-851

Oishi Y, Spann NJ, Link VM, Muse ED, Strid T, Edillor C, Kolar MJ, Matsuzaka T, Hayakawa S, Tao J, Kaikkonen MU, Carlin AF, Lam MT, Manabe I, Shimano H, Saghatelian A \& Glass CK 2017 SREBP1 contributes to resolution of pro-inflammatory TLR4 signaling by reprogramming fatty acid metabolism. Cell Metabolism 25 412-427

Rincon G, Islastrejo A, Castillo AR, Bauman DE, German BJ \& Medrano JF 2012 Polymorphisms in genes in the SREBP1 signalling pathway and SCD are associated with milk fatty acid composition in Holstein cattle. Journal of Dairy Research 79 66-75

Shi X, Li D, Deng Q, Peng Z, Zhao C, Li X, Wang Z, Li X \& Liu G 2016 Acetoacetic acid induces oxidative stress to inhibit the assembly of very low density lipoprotein in bovine hepatocytes. Journal of Dairy Research 83 442-446

Song Y, Li N, Gu J, Fu S, Peng Z, Zhao C, Zhang Y, Li X, Wang Z, Li X \& Liu G $2016 \beta$-Hydroxybutyrate induces bovine hepatocyte apoptosis via an ROS-p38 signaling pathway. Journal of Dairy Science 99 9184-9198

Spann NJ, Garmire LX, Mcdonald JG, Myers DS, Milne SB, Shibata N, Reichart D, Fox JN, Shaked I, Heudobler D, Raetz CR, Wang EW, Kelly SL, Sullards MC, Murphy RC, Merrill AH, Jr, Brown HA, Dennis EA, Li AC, Ley K, Tsimikas S, Fahy E, Subramaniam S, Quehenberger O, Russell DW \& Glass CK 2012 Regulated accumulation of desmosterol integrates macrophage lipid metabolism and inflammatory responses. Cell 151 138-152 
Staniewski B, Kielczewska K, Smoczyński M, Baranowska M, Czerniewicz M \& Brandt W 2012 Effect of high pressures on the composition of milk fat triacylglycerols. Milchwissenschaft-milk Science International 67 18-21

Wei S \& Espenshade PJ 2012 Expanding roles for SREBP in metabolism. Cell Metabolism 16 414-419

Wu Q, Liu MC, Yang J, Wang JF \& Zhu YH 2015 Lactobacillus rhamnosus GR-1 ameliorates Escherichia coli-induced inflammation and cell damage via attenuation of ASC-independent NLRP3 inflammasome activation. Applied and Environmental Microbiology 82 1173-1182
Xu DD, Wang G, He XJ, Wang JF, Yang B, Sun ZP, Sun DB, He QY, Zhang X \& Wu R 2017 17ß-Estradiol and progesterone decrease MDP induced NOD2 expression in bovine mammary epithelial cells. Veterinary Immunology Immunopathology 188 59-64

Zhang C, Shin DJ \& Osborne TF 2005 A simple promoter containing two Sp1 sites controls the expression of sterol-regulatory-element-binding protein 1a (SREBP-1a). Biochemical Journal 386 161-168

Zhang M, Zhang S, Hui Q, Lei L, Du X, Gao W, Zhang R, Liu G, Li X \& Li X $2015 \beta$-Hydroxybutyrate facilitates fatty acids synthesis mediated by sterol regulatory element-binding protein1 in bovine mammary epithelial cells. Cellular Physiology and Biochemistry 37 2115-2124 